\title{
High-resolution Catheters for Arrhythmic Driver Detection: Preliminary Results in Atrial Fibrillation
}

\author{
Alice Andalò ${ }^{1}$, Giuseppe Calamia ${ }^{1}$, Claudio Fabbri ${ }^{1}$, Paolo Sabbatani ${ }^{2}$, Michele Monaci ${ }^{1}$, Stefano \\ Severi ${ }^{1}$, Cristiana Corsi ${ }^{1}$ \\ ${ }^{1}$ DEI, Campus of Cesena, University of Bologna, Cesena, Italy \\ ${ }^{2}$ Bufalini Hospital, AUSL della Romagna, Cesena, Italy
}

\begin{abstract}
Mechanisms that sustain atrial fibrillation $(A F)$ are not yet clearly identified. Recently, there has been an increasing interest in detecting spiral waves and rotors and in understanding how these potential drivers sustaining $A F$ might be therapeutic targets for catheter-based ablation. The aim of this study was to understand if arrhythmic drivers may be detected using electrograms (EGMs) acquired with a new high-resolution catheter.

Spatiotemporal organization of atrial fibrillation was studied applying a previously developed and validated algorithm based on phase analysis for local activation timings (LATs) detection and on the persistence of phase singularities for meandering and stable rotor identification on the left atrium (LA) wall.

On in vivo data, we analysed 28 segments of ten second duration in three patients. Six stable rotors were identified; two rotors had a persistence of 10 seconds; the remaining 4 rotors were shorter (mean persistence in time: 2.91 $\pm 3.27 \mathrm{~s}$ ). Results show the proposed analysis is feasible and processing of EGMs acquired with high-resolution catheters allows detection of stable rotors. Probably due to low spatial coverage of the catheter, meandering rotors were not tracked. These results need confirmation on a larger dataset and hold promise for a comprehensive arrhythmic driver detection with different mapping catheters.
\end{abstract}

\section{Introduction}

Atrial fibrillation (AF) is one of the most common cardiac arrhythmia worldwide and its presence is associated with high rates of morbidity and mortality. In addition to drug therapy, catheter ablation provides the most effective treatment option to restore sinus rhythm by eliminating drivers triggering or sustaining AF. [1]

Mechanisms that sustain AF are not yet clearly identified (Figure 1). Pulmonary vein isolation (PVI) is the most important strategy for the management of paroxys-
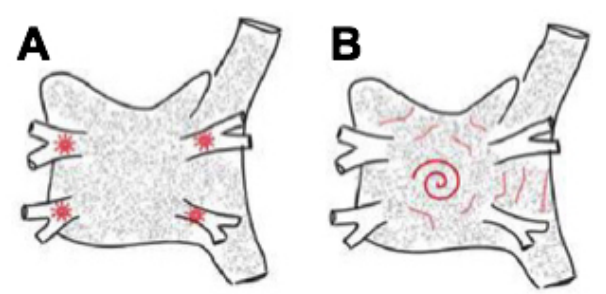

Figure 1. Different AF mechanisms: ectopic foci located in PVs (A) and spiral waves (B). Adapted from [4]

mal atrial fibrillation, due to the presence in this area of a focal source that can trigger AF [2]. Nevertheless, the isolation of the pulmonary veins alone is not enough to prevent recurrences in the long term: success rates vary between $60 \%$ to $80 \%$ for paroxysmal $\mathrm{AF}$ and between $50 \%$ to $60 \%$ for persistent $\mathrm{AF}$ [3].

Recent studies pointed out that persistent AF is sustained by further mechanism known as reentrant waves (rotors). The removal of this reentry could lead to a better procedural outcome than PVI alone. Therefore, the correct identification of both presence and location of rotors during the ablation procedure could support the physician to understand which patient-specific mechanisms are responsible for AF and consequently optimize therapy administration.

The aim of this study was to understand if arrhythmic drivers may be detected using electrograms (EGMs) acquired with a new high-resolution catheter.

\section{Materials and Methods}

\subsection{Clinical Data}

Data from three patients with persistent AF were acquired in electrophysiology (EP) lab at M. Bufalini Hospital, Cesena, Italy. All patients underwent an ablation procedure and the new Advisor ${ }^{\mathrm{TM}}$ HD Grid (Abbott Technologies) mapping catheter was used to acquire unipolar 


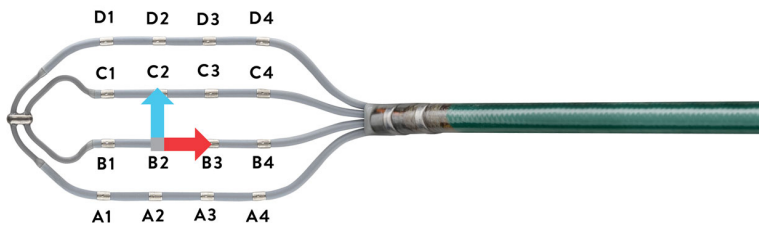

Figure 2. The Advisor ${ }^{\mathrm{TM}}$ HD Grid high resolution mapping catheter.

\section{EGMs.}

The Advisor ${ }^{\mathrm{TM}}$ HD Grid (Figure 2) is a high-resolution mapping catheter compound by 16 equidistant electrodes arranged in a $4 \times 4$ grid pattern, with an intra-electrodes spacing of $3 \mathrm{~mm}$. The catheter coverage area is equal to $81 \mathrm{~mm}^{2}$. This catheter is the first of its kind designed to facilitate substrate mapping and to account for directionality by recording the signals both along and through the splines.

\subsection{Data processing}

Spatiotemporal organization of atrial fibrillation was studied applying a previously developed and validated algorithm [5] based on phase analysis for local atrial activation timings (LAATs) detection and on the persistence of phase singularities for meandering and stable rotor identification on the left atrium (LA) wall.

After filtering the EGM signals and removing the ventricular far field, we estimated the dominant frequency of the signal according to the Botteron's approach, and to detect LAAT we used a modified version of the electrogram recomposition from sinusoidal wavelets proposed by Kuklik [6] (Figure 3).

Once the LAATs were detected, 3D phase maps were reconstructed on the area of the 3D LA anatomical surface mesh covered by the catheter. To each point on the anatomy was assigned the phase value computed considering the signal from the nearest electrode.

The rotor detection algorithm is based on the investigation of the phase singularity (PS) points. A PS is a point surrounded by a gradual phase transition between neighbouring points up to an inversion phase (from $\pi$ to $-\pi$ ), as described by Equation 1 where $\mathrm{c}$ is a closed loop surrounding the given point, $\phi$ is the phase value in space, $\nabla$ is a spatial derivative.

$$
\frac{1}{2 \pi} \oint_{c} \nabla \phi d r
$$

Since we have a discrete mesh of electrodes, the integral has to be discretized and approximated. To each region

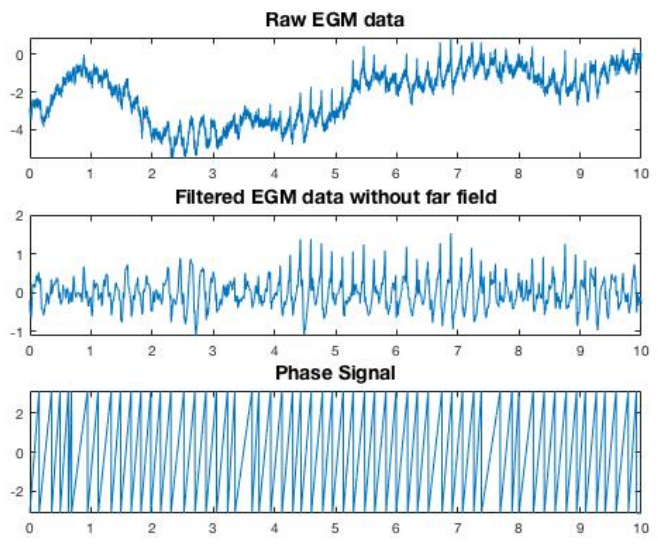

Figure 3. An example of raw EGM (top panel), EGM after filtering and far-field removal (central panel), resulting phase signal (bottom panel)

of the LA mesh, the neighboring electrodes were assessed and the phase gradient between them was computed $(\Delta \phi)$. If the region under investigation is surrounded by a gradual phase transition between its neighboring regions plus an inversion phase, the midpoint of the centroids of the regions represents a PS point.

Since, for each time frame, the algorithm might detect several PS points due to the discretization, a PS point was defined as the pivot of a rotor if it persists over time. For the correct evaluation of the persistence of the PS two parameters taking into account spatial and temporal allowed gaps have been set.

In addition, the rotor was defined stable in the space if the coordinates of its pivot remains inside a region of a certain radius throughout its lifecycle. Otherwise, the rotor was defined meandering or mobile.

Finally, only the phase singularity that persisted, within the region covered by the catheter, for a duration greater than twice the mean dominant period (defined as the inverse of the dominant frequency), is identified as rotor.

The validation of the algorithm for the identification of PS was performed in 2D; this simplification has also been possible considering the simple and regular geometry of the catheter under investigation.

The algorithm for the evaluation of rotor detection and persistence was validated using simulated rotor data. A code that generated the artificial PS belonging to different rotors of distinct duration was implemented. The entire workflow was also tested with EGMs acquired artificially from a simulated spiral wave propagation in 2D tissue.

We analysed 28 segments of ten second duration in three patients, divided as follows:

- Patient \#1: 4 segments

- Patient \#2: 15 segments 
- Patient \#3: 9 segments

During the ablation procedure the physician tries to acquire signals in the regions where scientific studies show a higher probability of detecting spiral patterns (roof, posterior wall, regions close to the ostia of the PVs and the region between the auricle and the left PV ostium). However, they are often not easily reachable with the catheter, or the physician cannot maintains an adequate stability of the catheter in the desired region and this justifies the different number of segments acquired for each study.

\section{Results}

On the simulated data, identification of synthetic rotors was correct in $100 \%$ of the tested cases.

On in vivo data, the identified rotors location and duration are summarize in Table 1, while an example of 3D phase map is reported in Figure 4. From the analysis we have identified six stable rotors. Two rotors had a persistence of 10 seconds while the remaining 4 rotors were shorter and on average have a duration of $2.91 \mathrm{~s}$. It can be noted that the positions in which the rotors have been identified are those indicated by scientific studies as regions where it is more common to find spiral patterns.

Table 1. Identified Rotors

\begin{tabular}{ccc}
\hline \hline Patient & Segment Position & Duration [s] \\
\hline$\# 1$ & roof & $1.06 \mathrm{~s}$ \\
$\# 2$ & anterior wall, near auricola & $10 \mathrm{~s}$ \\
$\# 2$ & wall between right PVs & $1.59 \mathrm{~s}$ \\
$\# 2$ & floor right IPV & $7.81 \mathrm{~s}$ \\
$\# 3$ & anterior wall & $10 \mathrm{~s}$ \\
$\# 3$ & left side, near PVs & $1.18 \mathrm{~s}$ \\
\hline \hline
\end{tabular}

\section{Discussion and Conclusions}

This is the first study evaluating the presence of rotors with the high-resolution Advisor ${ }^{\mathrm{TM}}$ HD Grid catheter.

From the processing of all the signals acquired by the 3 patients, stable rotors which lasted for the entire acquisition (10 s) were identified in 2 of the 3 patients.

The remaining 4 shorter rotors might be only a portion of the mobile rotor that has passed through the area covered by the catheter.

In this study, it was possible to evaluate only stable rotors. In fact, the limited coverage of the atrium surface, imposed by the small size of the Advisor ${ }^{\mathrm{TM}} \mathrm{HD}$ Grid catheter does not allow to cover large regions of the atrium to justify the search for meandering rotors.

The presented workflow was also tested on in vivo data acquired from different catheters where also meandering

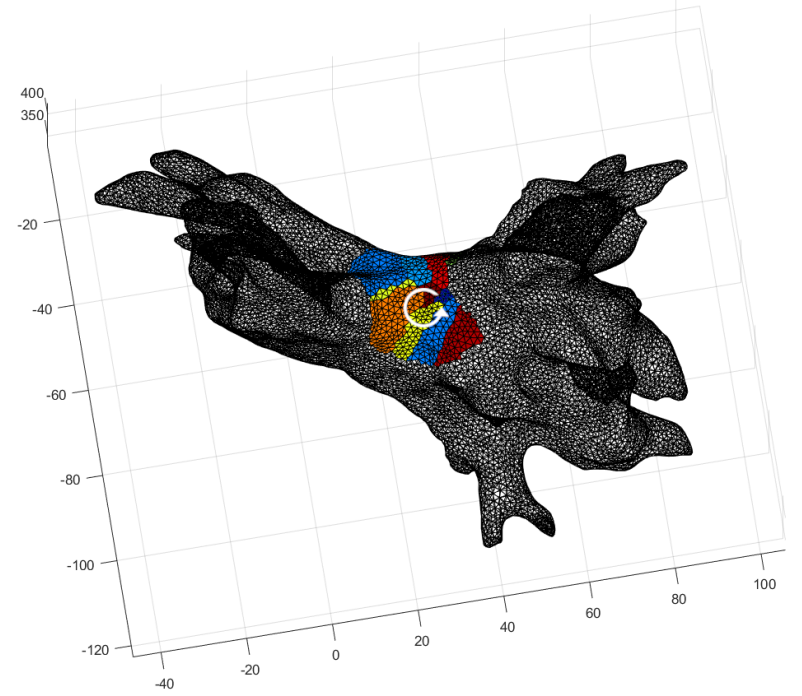

Figure 4. 3D phase map from EGMs acquired with a high-resolution mapping catheter, superimposed to the patient-specific anatomical model; the white arrow shows the anatomical regions around a phase singularity point.

rotors were found, probably due to the greater coverage of the catheter used.

These results, supported by the high resolution of data acquisition, if confirmed by analyzing a larger and possibly multicentric datset, could validate the theory of rotors as a mechanism for the genesis and maintenance of AF. In fact, the existence of rotors is subject of debate in the scientific community and some argue that they are due to artifacts caused by low resolution catheters. Identification of the rotors performed using data acquired with high resolution catheters would support their existence.

The results of this work represent a starting point for numerous other studies of considerable interest due to their potential impact in clinical practice. A possible future development of this project could be to consider different diagnostic catheters and verify how much and in which way the catheter can influence the detection of the rotors.

Moreover, there may be a chance to export this method in clinical practice, in order to be able to give to the clinicians a useful tool that can help during the $\mathrm{AF}$ ablation procedures performed with electroanatomical mapping systems. This system of automatic analysis of endocavitary data could be used during the ablation procedure, providing in real-time the information on both the identification of phase singularities on the 3D map and their persistence. This could give the physician the possibility of identifying the rotor center as an ablative target, to improve effectiveness of the treatment of patients suffering from persistent AF.

In conclusion, these preliminary data show that it is pos- 
sible to identify and locate rotors and this may help clinicians to optimize therapy administration removing the additional drivers that sustain the arrhythmia.

\section{References}

[1] Calkins H, et al. 2017HRS/EHRA/ECAS/APHRS/SOLAECE expert consensus statement on catheter and surgical ablation of atrial fibrillation: Executive summary. EP Europace 09 2017;20(1):157-208. ISSN 1099-5129.

[2] Hassaguerre M, Jas P, Shah DC, Takahashi A, Hocini M, Quiniou G, Garrigue S, Le Mouroux A, Le Mtayer P, Clmenty J. Spontaneous initiation of atrial fibrillation by ectopic beats originating in the pulmonary veins. New England Journal of Medicine 1998;339(10):659-666. PMID: 9725923.

[3] Ganesan AN, Shipp NJ, Brooks AG, Kuklik P, Lau DH, Lim HS, Sullivan T, RobertsThomson KC, Sanders P. Long term outcomes of catheter ablation of atrial fibrillation: A systematic review and meta analysis. Journal of the American Heart Association 2013;2(2):e004549.

[4] A B Zaman Ma Bm BChir J, Schricker Md A, G Lalani Md G, Trikha Bs R, E Krummen Md D, M Narayan Md PhD $\mathrm{S}$. Focal impulse and rotor mapping (firm): Conceptualizing and treating atrial fibrillation. Journal of atrial fibrillation 2014;7(2):1103. ISSN 1941-6911.

[5] Valinoti M, Berto F, Alessandrini M, Mantovan R, Loewe A, Dssel O, Severi S, Corsi C. Phase analysis of endoatrial electrograms for $3 \mathrm{~d}$ rotor detection in atrial fibrillation. In 2017 Computing in Cardiology (CinC). 2017; 1-4.

[6] Kuklik P, Zeemering S, Maesen B, Maessen J, Crijns HJ, Verheule S, Ganesan AN, Schotten U. Reconstruction of instantaneous phase of unipolar atrial contact electrogram using a concept of sinusoidal recomposition and hilbert transform. IEEE Transactions on Biomedical Engineering 2015; 62(1):296-302.

Address for correspondence:

Alice Andalò

Department of Electric, Electronic and Information Engineering Guglielmo Marconi DEI, University of Bologna,

Via dell Università 50, 47521 - Cesena (FC)

alice.andalo2@unibo.it 